\title{
Decision-Making Optimization of Mine Gas Monitoring Based on Gauss Process Regression and Interval Number Correlation Analysis
}

\author{
Dingwen Dong (iD \\ School of Safety Science and Engineering, Xi'an University of Science and Technology, Xi'an 710054, Shaanxi, China \\ Correspondence should be addressed to Dingwen Dong; dingw_dong@163.com
}

Received 19 April 2021; Revised 6 October 2021; Accepted 20 October 2021; Published 9 November 2021

Academic Editor: Kamal Kumar

Copyright (c) 2021 Dingwen Dong. This is an open access article distributed under the Creative Commons Attribution License, which permits unrestricted use, distribution, and reproduction in any medium, provided the original work is properly cited.

\begin{abstract}
For the subjective limitation of gas sensor calibration in coal mines, a decision-making method for gas sensor calibration under monitoring failure was studied based on the Gauss process regression (GPR) and the correlation analysis of interval numbers. Based on the correlation characteristics of gas monitoring data of each monitoring point in the work face area in coal mine, the initial confidence interval of gas concentration in monitoring failure period was obtained by GPR, and then the confidence interval was further optimized by the correlation analysis of interval numbers. According to the correlation characteristics of monitoring data of each monitoring point, its similarity of dynamic variation tendency was measured by using Euclidean distance of interval numbers, and the optimal confidence interval was determined by calculating the correlation degree of interval numbers. The case study shows that making full use of the effective monitoring information of multiple monitoring points ensures the reliability of the initial confidence interval; the dynamic adjustment of model parameters in correlation analysis of interval number avoids the subjectivity defect of similar methods and further obtains the consistency between interval numbers' reliability and correlation degree, which can ensure the effectiveness of the application of this method.
\end{abstract}

\section{Introduction}

Because of the harsh environment in underground coal mine, the gas concentration monitoring of sensor is affected by water vapor and dust, and its data transmission is also affected by the electromagnetic interference and so on, so the monitoring data of gas concentration often distort. Due to the fact that the tolerable error range of monitoring data is $5 \% \sim 15 \%$, in order to ensure reliable monitoring, the artificial sensor calibration is an important way. The present software of the main computer of safety monitoring and control system in coal mine only has the function of statistics and large number (beyond the range of gas concentration) filtering, but it lacks the decision-making support function of sensor calibration. Moreover, the variation of gas concentration is highly complex in the working face because the air flow pattern is so complex in this area, so that the technicians of sensor calibration are limited by subjective experience and lack objective scientific basis. Therefore, the basic data set is constructed through getting the large data of gas concentration monitoring online, and the decision-making support method of gas sensor calibration is researched, and then it is integrated in the portable devices and helps technicians in sensor calibration based on the Internet of things technology; it will greatly improve the reliability and effectiveness of sensor calibration. At present, there is a lack of practical research findings in this research field to meet the application needs in coal mines in China. So it is of great practical significance to research a scientific and reasonable decisionmaking support method for gas sensor calibration, which will play an important role in safety monitoring and prevention of gas explosion accident in coal mines.

Based on the actual needs of sensor calibration decisionmaking in coal mines in China, the motivation of this paper is to provide a reliable and quantitative method for sensor calibration to make up for the defect of relying only on subjective judgment; it is studied by processing gas monitoring data combined with GPR and interval number 
correlation analysis. The GPR has the advantage of selfadaptive optimization of parameter estimation in its training process and outputting the prediction interval at a certain confidence level; it is suitable for dealing with high dimensional and nonlinear complex problems $[1,2]$ and achieved good application effects in pattern recognition of complex network systems and nonlinear systems [3, 4], regression prediction and classification [5-9], and so forth. So it can be applied for the gas concentration prediction while monitoring failure of sensor and obtain a confidence interval in the form of normal distribution interval number based on a certain confidence level; it can be used as the initial interval for decision-making. Due to the uncertainty and complexity of gas concentration variation, as well as the limitations of technicians' subjective judgment, given a specific value to guide the sensor calibration, there may occur large deviation. Therefore, this paper uses interval number to study the decision-making optimization of mine gas monitoring. The prediction interval may become larger when modeling the gas concentration data from the monitoring points because of the complexity of gas flow in air flow disorder area (such as the working face). For technicians, the decision-making interval of sensor calibration not only requires high confidence but also is meaningless if the interval length is too large, so it may be necessary to further optimize the initial decision-making interval, and the innovation of this paper is to solve the problems. As a method of uncertainty analysis, the interval number calculation has also achieved good effects in optimization solution [10-13], risk analysis and project evaluation [14-16], multiattribute and group decision-making problems [17-19], and so forth. Because it is impossible to determine the influence degree of various influencing factors on gas concentration, it can only make use of the structural characteristics of ventilation roadway according to the gas flow law and fully mine the useful information in monitoring data to provide the optimal decision-making basis. The normal distribution interval numbers from GPR prediction have good statistical characteristics, and the correlation analysis model can be established by fusing the monitoring data of multiple relevant monitoring points; the correlation degree of interval number will be consistent with the reliability of optimization results; this advantage is used to dynamically adjust the model parameters and obtain a confidence interval with small interval length and higher reliably, which will provide good decision-making support for gas sensor calibration in gas monitoring management in coal mines.

Based on the above analysis, according to the physical characteristics of gas flow in ventilation roadway and the good statistical properties of the prediction results of GPR, the data set is constructed to make full use of the correlation characteristics of the gas monitoring data at the different monitoring points, the measurement of the correlation analysis of interval numbers was defined, and the method of the correlation analysis of interval numbers was used to further optimize the gas concentration prediction interval, so as to meet the practical application requirements of sensor calibration in the case of monitoring failure.

\section{GPR Prediction Model of Gas Concentration}

2.1. The Correlation Characteristics Analysis of Gas Concentration Monitoring Data. The gas monitoring of working face area is the most important in coal mines, and reliable monitoring of this area is the key to safety control. Therefore, this paper mainly studies the method for decision-making optimization of gas concentration monitoring at the working face monitoring point, and the other monitoring points in the working face area are deemed as associated monitoring points.

The layout of gas monitoring points in the working face area is shown in Figure 1. According to the principle of mass conservation, the gas flow quantity in the air intake flow plus the gas emission from the working face and the goaf is equal to the total gas flow quantity in the air return flow. The small arrow in Figure 1 shows the direction of gas flow in the ventilation roadways, and the big arrow shows the direction of the mining. $T_{\text {in }}, T_{u}, T_{w}, T_{r}$ are used to express four gas monitoring points in the working face area: the air intake roadway, the upper corner, the working face, and the air return roadway. The monitoring point $T_{w}$ is set in the air return roadway because of the complex air flow pattern in the working face, the gas concentration varies dynamically in the direction of the air flow and the direction of the mining, and the gas flow can mix evenly in the air flow when arriving at position $T_{w}$, so the gas concentration value at the working face monitoring point can be regarded as the average value of the gas concentration in the working face area. The variation trends of gas concentration at monitoring points $T_{w}$ and $T_{r}$ are similar to that at monitoring point $T_{u}$, because the air flow is high disorder at upper corner, so the variation characteristics of gas concentration are the most complex. In addition, the gas concentration value at monitoring point $T_{\text {in }}$ is smaller. The time series formed by gas concentration monitoring data at each monitoring point is expressed by $C_{\mathrm{in}}, C_{u}, C_{w}, C_{r}$, which formed a multivariable time series data set.

The theoretical characteristics of the gas concentration distribution at each monitoring point in the working face area are the basis of decision-making optimization under the condition of monitoring failure. Therefore, through the fusion analysis of time series formed by the gas concentration monitoring data at each monitoring point in the working face area, a reasonable confidence interval can be obtained by the means of large data processing to guide the gas sensor calibration.

2.2. GPR Prediction Model. According to the correlation characteristics analysis of gas concentration monitoring data in the working face area, based on the multivariable time series formed by real-time monitoring data of each monitoring point, the method of calculating initial confidence 


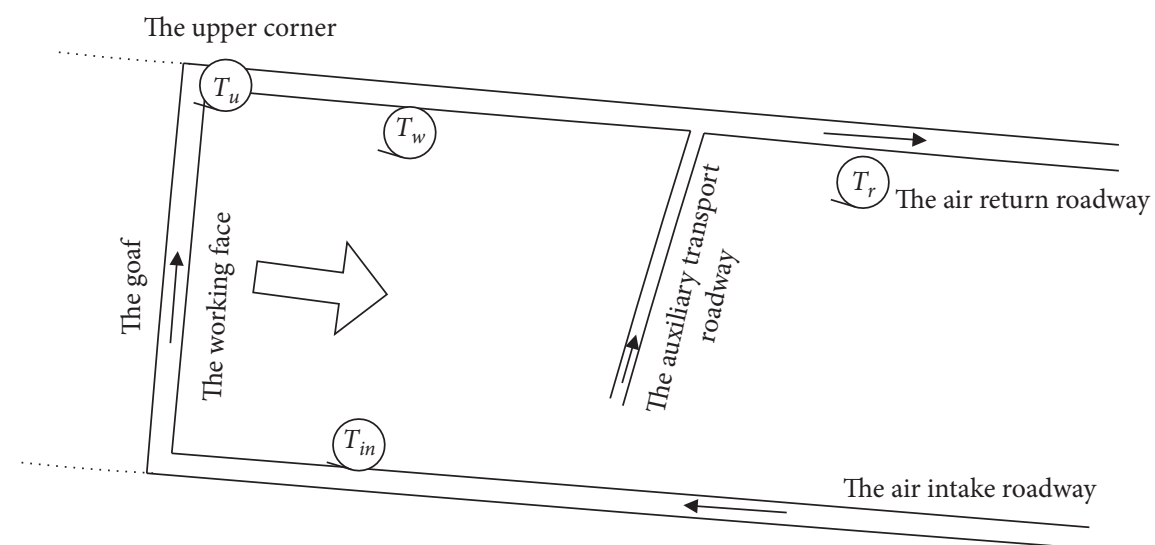

Figure 1: Layout of gas monitoring points in the working face area.

interval of gas concentration at the working face monitoring point by GPR is as follows, and its calculation process is shown in Figure 2.

\subsubsection{Determining the Basic Dataset for the GPR Prediction} Model. In the calculation process of the decision-making optimization, it is necessary to consider that the monitoring failure may occur at the associated monitoring points at the same time. The multivariable data sets are the basic data for gas concentration prediction; therefore, the prediction calculation under the monitoring failure at all monitoring points in the working face area must be considered.

$C$ is used to express the basic data set formed by the time series of multivariable gas concentration monitoring data. According to the monitoring failure at different monitoring points, the initial confidence interval of gas concentration at $T_{\text {in }}$ is calculated based on the correlation characteristics analysis of gas concentration monitoring data in the working face area, $C=\left\{C_{\text {in }}, C_{r}\right\}$; when calculating initial confidence interval of gas concentration at $T_{u}, C=\left\{C_{\mathrm{in}}, C_{u}\right\}$; when calculating initial confidence interval of gas concentration at $T_{w}, C=\left\{C_{\text {in }}, C_{u}, C_{w}\right\}$; when calculating initial confidence interval of gas concentration at $T_{r}, C=\left\{C_{\text {in }}, C_{u}, C_{w}, C_{r}\right\}$. If one associated monitoring point fails, the variable should be reduced; and if all the associated monitoring points fail, an autoregressive prediction model of gas concentration will be established.

2.2.2. GPR Model Training. Because the gas concentration monitoring data belongs to high-frequency time series, the time point for decision-making calculation is set to $t$, and $C$ will be expressed as $C(t)$, and the time series of gas concentration monitoring data corresponding to each monitoring point will be expressed as $C_{\text {in }}(t), C_{u}(t), C_{w}(t), C_{r}(t)$. According to the Gauss process regression theory, it is able to get a prediction value $\hat{y}$ and its confidence interval $\hat{y} \in\left[\hat{y}_{l}, \hat{y}_{u}\right]$ by training the regression model GP $(X, y)$, where $X$ is the $N \times n$ input matrix, $y$ is the $n$-dimension output vector, the samples $\left(x_{i}, y_{i}\right) \mid i=1, \ldots n$ are $n$ samples in $C, N=1,2,3,4$, and then $\left[\widehat{y}_{l}, \widehat{y}_{u}\right]$ is the initial confidence

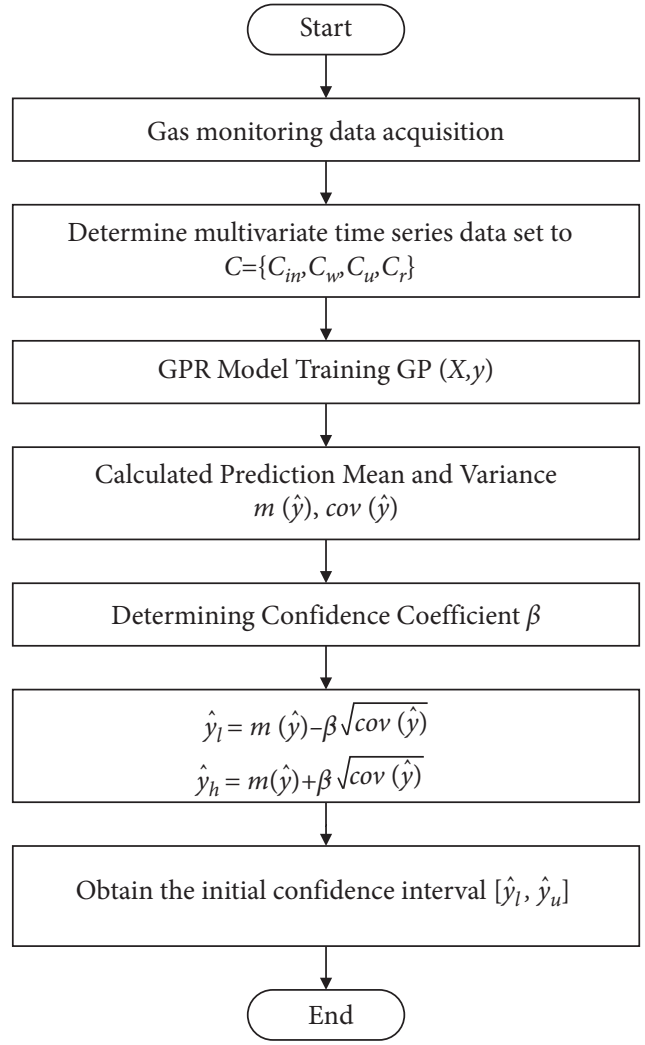

FIGURE 2: Calculation process of the GPR prediction model for gas concentration.

interval of gas concentration; the calculation model can be seen as a regression problem:

$$
y=f(x)+\varepsilon,
$$

where $\varepsilon$ obeys the probability distribution $N\left(0, \sigma_{n}^{2}\right)$ and is noise, and the prior distribution of $y$ is

$$
y \mid X, K \sim N\left(0, K(X, X)+\sigma_{n}^{2} I\right),
$$

where $K(X, X)$ is the covariance matrix, in which each $k(X, X)$ is covariance function or kernel function. For a series of test input samples $X_{*}$, the joint probability distribution of its target value and function values is as follows: 


$$
\left[\begin{array}{l}
y \\
\hat{y}
\end{array}\right] \sim N\left(0,\left[\begin{array}{cc}
K(X, X)+\sigma_{n}^{2} I & K\left(X, X_{*}\right) \\
K\left(X_{*}, X\right) & K\left(X_{*}, X_{*}\right)
\end{array}\right]\right) .
$$

$K=K(X, X)$ and $K_{*}=K\left(X_{*}, X_{*}\right)$ are set; if there is only a test sample, $k_{*}=k\left(x_{*}\right)$ is used to represent the covariance between test sample and $n$ training sample; the Gaussian process regression prediction can be expressed as follows:

$$
\begin{aligned}
\hat{y} \mid X, y, X_{*} & \sim N(m(\hat{y}), \operatorname{cov}(\hat{y})), \\
m(\hat{y}) & =k_{*}^{T}\left(K+\sigma_{n}^{2} I\right)^{-1} y, \\
\operatorname{cov}(\widehat{y}) & =k\left(x, x_{*}\right)-k_{*}^{T}\left(K+\sigma_{n}^{2} I\right)^{-1} k_{*},
\end{aligned}
$$

where $m(\hat{y})$ is the mean value function, $\operatorname{cov}(\hat{y})$ is the variance function, $\sigma^{n}$ is the sequence variance, $I$ is the unit matrix, and $T$ expresses matrix transposition. The kernel function of Gaussian process model is as follows because it can be parameterized in terms of hyperparameters.

$$
k\left(x_{p}, x_{q}\right)=\sigma_{f}^{2} \exp \left(-\frac{\left(x_{p}-x_{q}\right)^{2}}{2 l^{2}}\right)+\sigma_{n}^{2} \delta_{p q},
$$

where $\_\delta_{p q}$ is a Kronecker delta which is one if $p=q$ and zero otherwise; $\theta=\left\{l, \sigma_{f}^{2}, \sigma_{n}^{2}\right\}$ is a vector of hyperparameters, and it is directly related to the training process of the model, so the values of hyperparameters will affect the prediction accuracy. In the training phase, it is completed by bringing into the gas concentration monitoring data before time point $t-1$ (contains $t-1$ ) of each monitoring point as input sample and expected output.

2.2.3. GPR Prediction. The gas concentration monitoring data vectors of each associated monitoring points at time $t$ are input into the prediction model completed by training, and $m(\hat{y})$ and $\operatorname{cov}(\hat{y})$ are obtained to get the initial confidence interval $\left[\hat{y}_{l}, \hat{y}_{u}\right]$ for the different monitoring points.

\subsubsection{Determining the Initial Confidence Interval.} According to the characteristics of normal distribution, the calculation formula of confidence interval is $[m(\hat{y})-\beta \sqrt{\operatorname{cov}(\hat{y})}, m(\hat{y})+\beta \sqrt{\operatorname{cov}(\hat{y})}]$, where $\beta$ is confidence coefficient, and it represents the confidence level. For monitoring point $T_{\text {in }}$, the smaller gas flow quantity enters the working face with the air flow, while when arriving at $T$, the gas flow has been fully mixed in the air flow, and the air flow pattern is relatively single, so the initial confidence interval length of the gas concentration at these two monitoring points can be smaller, which helps to reduce the subsequent calculation's complexity; the confidence level is taken as $95.45 \% ; \beta=2$ for monitoring point $T_{u}$, due to the complexity of the air flow pattern and the gas emission from the goaf to the working face being uncertain; meanwhile, the monitoring point $T_{w}$ is arranged in the air return roadway; moreover, the influence of the production process is more complex, so the confidence level is taken as $99.73 \%$, and $\beta=$ 3 in these two monitoring points.

\section{Confidence Interval Optimization of Gas Concentration}

The initial confidence interval of gas concentration obtained by GPR prediction is expressed as $\hat{y}_{0}=\left[\hat{y}_{0 l}, \hat{y}_{0 u}\right]$ in this section; considering the practical application, the current monitoring period is $30 \mathrm{~s}$ in most of coal mines in China, and the three minutes are used for decision-making and manual calibration. Therefore, the interval number sequence $\hat{y}_{0}(j), j=1,2, \ldots, 6$, is formed by six consecutive calculations. For the optimization of the initial confidence interval, reducing the interval length on the premise of high reliability will improve the effectiveness of the application, which can be achieved by adjusting the confidence coefficient $\beta$, because the initial confidence interval is the normal distribution interval number. According to the characteristics of normal distribution, when $\beta=1$, the confidence level is $68.27 \%$, and when $\beta=3$, the confidence level is $99.73 \%$, so the step of $\beta$ is set as 0.1 to find the optimal interval when $\beta \in[1,3]$. While $y_{0}$ is shrinking, the confidence intervals of the other associated monitoring points will be constructed; when the gas monitoring of associated monitoring points is effective, the confidence intervals are obtained by statistical analysis, and when the gas monitoring fails, the GPR prediction model is constructed by using the basic data extraction method in Section 2.2 to calculate the confidence intervals, which are expressed as $y_{i}=\left[y_{i l}, y_{i u}\right]$, where $i=1,2,3$, representing the associated monitoring points $T_{\text {in }}, T_{u}, T_{r}$. The optimal confidence interval will be obtained by calculating the correlation degree of the gas concentration's confidence interval between the working face monitoring point and the associated monitoring points; the correlation coefficient of interval numbers is defined as

$$
r\left(\hat{y}_{0}(j), y_{i}(j)\right)=\frac{d_{\min }+\rho d_{\max }}{d_{0 i}(j)+\rho d_{\max }},
$$

where $d_{\max }=\max _{i} \max _{j} d_{0 i}(j), d_{\min }=\min _{i} \min _{j} d_{0 i}(j), \rho$ is the resolution coefficient, $\rho \in[0,1], \quad$ and $d_{0 i}(j)=d\left(\hat{y}_{0}(j), y_{i}(j)\right)$ is the Euclidean distance between $\hat{y}_{0}(j)$ and $y_{i}(j)$; its definition is as follows:

$$
d\left(\widehat{y}_{0}(j), y_{i}(j)\right)=\sqrt{\frac{\left(\hat{y}_{0 l}(j)-y_{i l}(j)\right)^{2}+\left(\hat{y}_{0 u}(j)-y_{i u}(j)\right)^{2}}{2}}
$$

and then the correlation degree of interval number is calculated as

$$
r\left(\hat{y}_{0}, y_{i}\right)=\frac{1}{3} \sum_{i=1}^{3} r\left(\hat{y}_{0}(j), y_{i}(j)\right) .
$$

Continually adjusting $\beta$ to obtain the correlation degree $r\left(\hat{y}_{0}, y_{i}\right)$, it reflects the correlation level of confidence interval in the gas concentration at the working face monitoring point and the associated monitoring points. When the correlation degree reaches its maximum $r$, the confidence interval of gas concentration will reach its optimal value $y_{*}$. The calculation flow of this model is shown in Figure 3. 


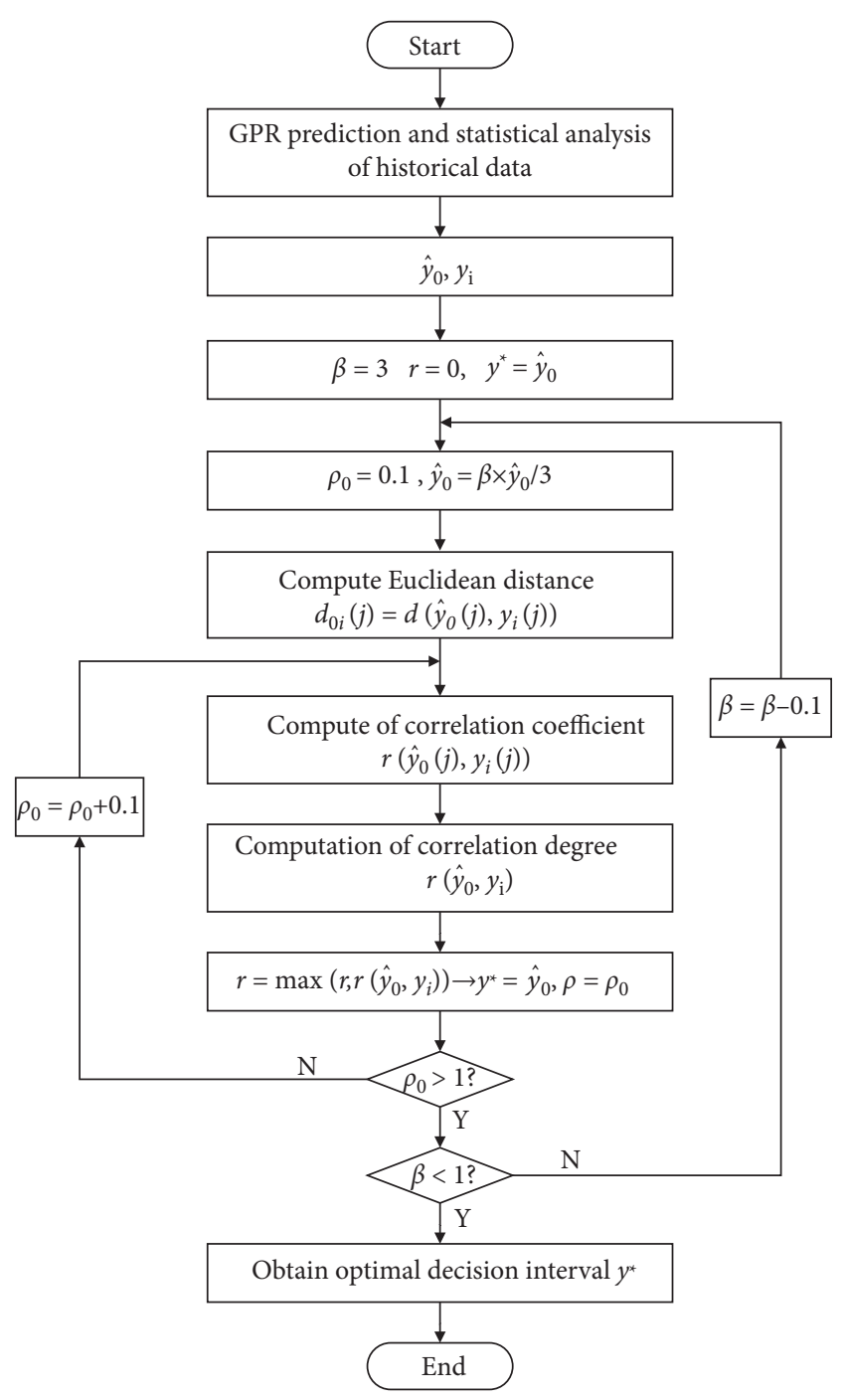

FIGURE 3: Calculation process of the interval number correlation analysis model.

Generally, the resolution coefficient $\rho$ in equation (6) is determined subjectively, but it directly affects the correlation degree, and it is the key to obtaining the optimal confidence interval, so it is determined dynamically and adaptively in this paper. According to the calculation flow in Figure 3, the historical data of a certain time span are abstracted to do correlation analysis, the resolution coefficient is set with the initial value $\rho_{0}=0.1$ and step size of 0.1 , and the resolution coefficient is continuously adjusted in the calculation process; when the correlation degree is maximum, the optimal resolution coefficient $\rho$ will be determined.

\section{Case Study}

Through the program development with MATLAB and online analysis with the safety monitoring and control system in a coal mine, the reliability, effectiveness, and the whole application process of this method are illustrated by comparing the actual monitoring values of gas concentration with the results of decision-making calculation.
4.1. The Correlation Characteristics Analysis of Gas Monitoring Data in the Working Face Area. In this paper, the time series of gas concentration monitoring data of four gas monitoring points in the working face area were collected from a coal mine in Huangling, Shaanxi Province in China; the basic data set was constituted from the data in December 2017; when the GPR model was trained, the choice of covariance function was in equation (5), the dimension of the input space was $1440^{*} 4$, and the one step continuous prediction was adopted. The sampling time is $1 \mathrm{~min}$, with a total of 1440 monitoring values in this time slot, and the statistical characteristics of the data are shown in Table 1; the safety regulations of coalmine industry in China stipulate that the gas concentration monitoring alarm value (upper limit) of working face is $1 \%$.

Limited by the page size, only the data samples used for result analysis on January 1, 2018, are shown in Figure 4. The correlation characteristics of the gas concentration's variation trend between the associated monitoring points and the working face monitoring point were illustrated.

It can be seen from Figure 4 that the dynamic variation trend of gas concentration at the working face monitoring point is similar to that in air return roadway; the variation trend of gas concentration at upper corner is also similar to that in air return roadway, but its gas concentration is higher as a whole; the gas concentration in intake roadway is smaller as a whole, and its correlation with other monitoring points is relatively weak. In addition, the variation trend of gas concentration is obviously affected by production alternation in a day. The first 8 hours (in $x$-axis 0 to 480 in Figure 4) witnessed no production because the work alternation institution of "two work shifts mining and one work shift preparation" has been adopted in this coal mine, so the gas concentration is smaller as a whole, and the gas concentration in the other 16 hours is bigger as a whole.

The following analysis is carried out by continuously calculating the confidence interval of gas concentration at the working face monitoring point and comparing it with the actual monitoring values.

4.2. The Results Analysis of GPR Prediction. After GPR prediction, the initial confidence interval of the gas concentration at the working face monitoring point on January 1, 2018, is obtained and shown in Figure 5; the three 8-hour results correspond to subgraphs (a), (b), and (c), respectively. The variation trends of the upper and lower limits of the initial confidence interval are basically consistent with the actual monitoring values. The actual monitoring values all fall in the $99.73 \%(\beta=3)$ confidence interval of GPR prediction, which indicates that the GPR prediction process regards the gas concentration time series of each monitoring point as a random process obeying normal distribution, and the correlation characteristics of the gas concentration monitoring data were fully used among each monitoring point to extract effective information, so that its calculation results have a higher reliability.

The reliability of the confidence interval is defined as the ratio between the number of actual monitoring values falling 
TABLE 1: Statistical characteristics of the data.

\begin{tabular}{lcccc}
\hline Monitoring points & The minimum (\%) & The maximum (\%) & The mean value (\%) & The upper limit (\%) \\
\hline$T_{j n}$ & 0.01 & 0.14 & 0.06 & 1.00 \\
$T_{w}$ & 0.06 & 0.78 & 0.21 & 1.00 \\
$T_{u}$ & 0.08 & 0.75 & 0.26 & 0.8 \\
$T_{r}$ & 0.09 & 0.79 & 0.24 & 1.00 \\
\hline
\end{tabular}

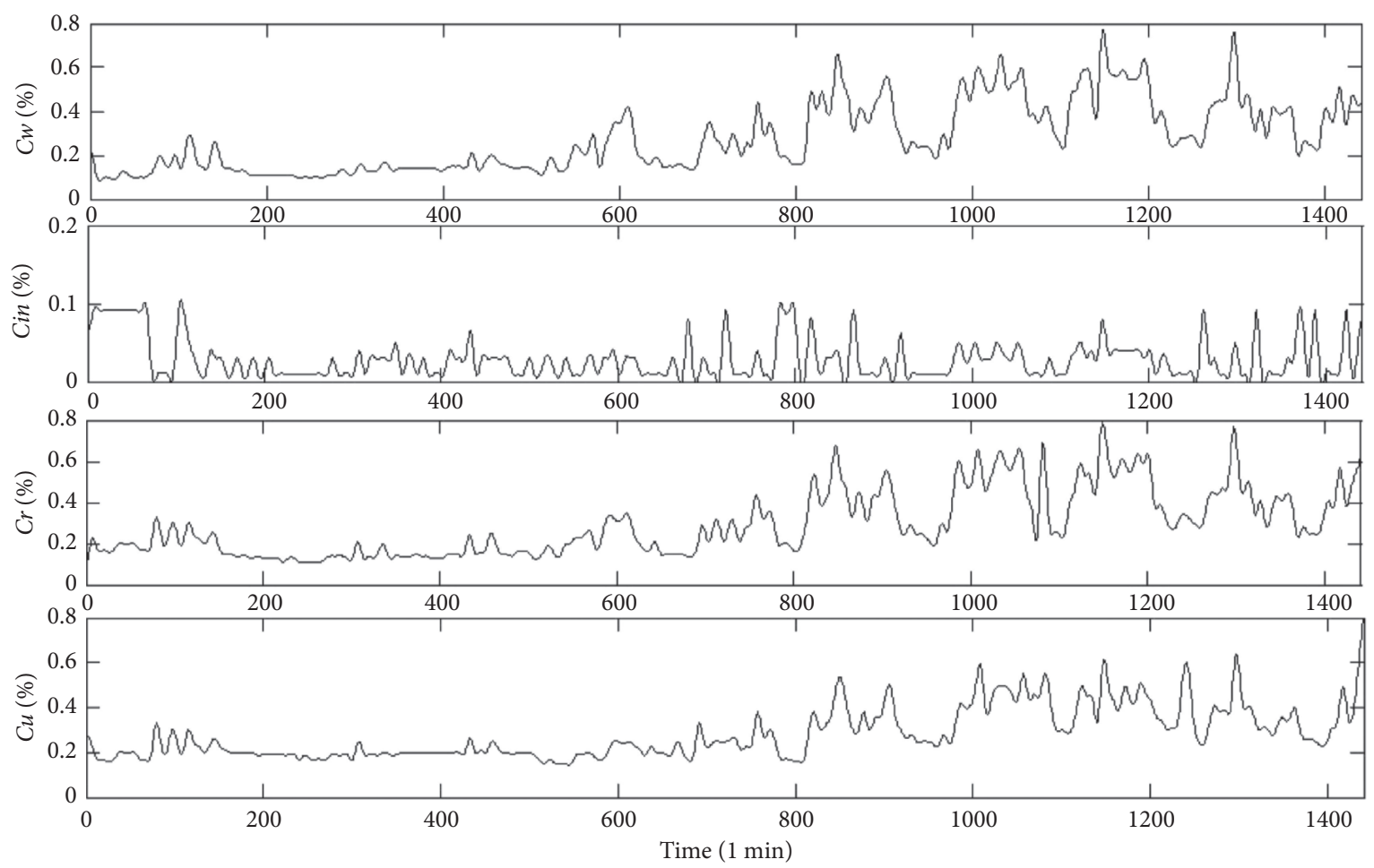

FIgURE 4: Time series of gas concentration at each monitoring point.

in the initial confidence interval and the prediction times. From the prediction results in Figure 5, it can be seen that the $99.73 \%(\beta=3)$ confidence interval contains all the actual monitoring values in this time period, so its reliability is $100 \%$, and the reliability of the initial confidence interval is ensured, but the average confidence interval length is $0.15 \%$. As the $68.27 \%$ confidence interval $(\beta=1)$, the variation trend of gas concentration in the first 8 hours (preparation shift) is gentle and reliable, and the actual monitoring values all fall within the initial confidence interval, but the variation trend of gas concentration in the other two 8 hours (production work shifts) is more complex; during this period, the actual monitoring values of 99 points fall outside the initial confidence interval, so its reliability is $89.68 \%$, while the average confidence interval length is $0.05 \%$. So the initial confidence interval length should be optimized to get an intuitive reference for technicians, and the optimal confidence interval should be obtained by adjusting the confidence coefficient $\beta$ dynamically.

4.3. Interval Number Correlation Analysis. While calculating the initial confidence interval of gas concentration dynamically, the correlation analysis of interval numbers is carried out according to the calculation method in Section 3. The GPR prediction model is trained by using the data of the first 11 months in 2017. In the process of confidence interval optimization for the data in December 2017, the resolution coefficient $\rho$ is adjusted continuously, and the correlation characteristics between the resolution coefficient $\rho$ and the average values of the confidence interval's reliability, the interval length, and the correlation degree are obtained and shown in Figure 6.

From the calculation results of historical data in Figure 6, it can be seen that the average correlation degree of confidence interval between the associated monitoring points and the working face monitoring point has a good relevance with the average reliability of confidence interval; their variation trends are consistent with the increase and decrease. Theoretically, there is a corresponding relationship between the reliability and the interval length; the interval length decreases with the decrease of the reliability, so the correlation degree of confidence interval is closely related to the interval length. The variation trend of the average correlation degree and the average reliability of confidence interval in Figure 6 reflects that the gas concentration monitoring data of the associated monitoring points contain the effective 

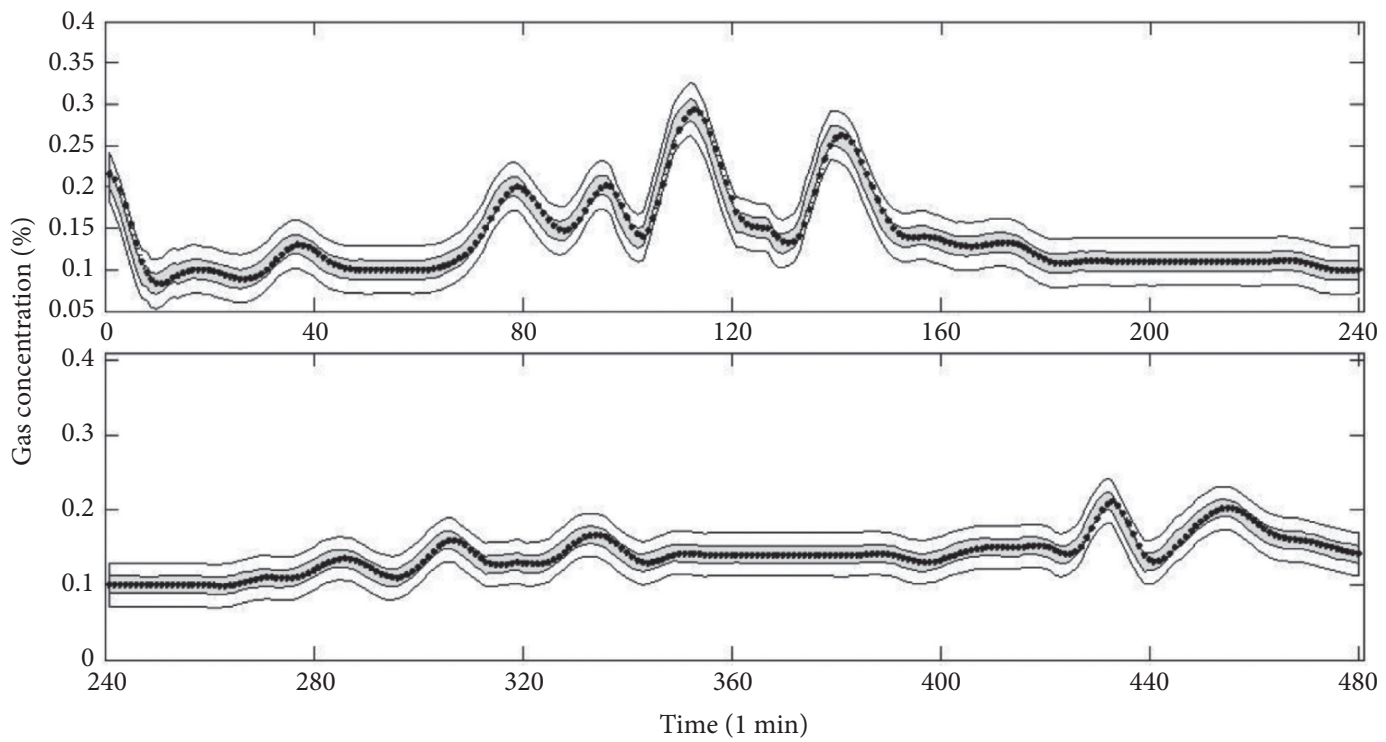

$\square 99.73 \%$ confidence interval

$\square 68.27 \%$ confidence interval

-. Actual monitoring value

(a)

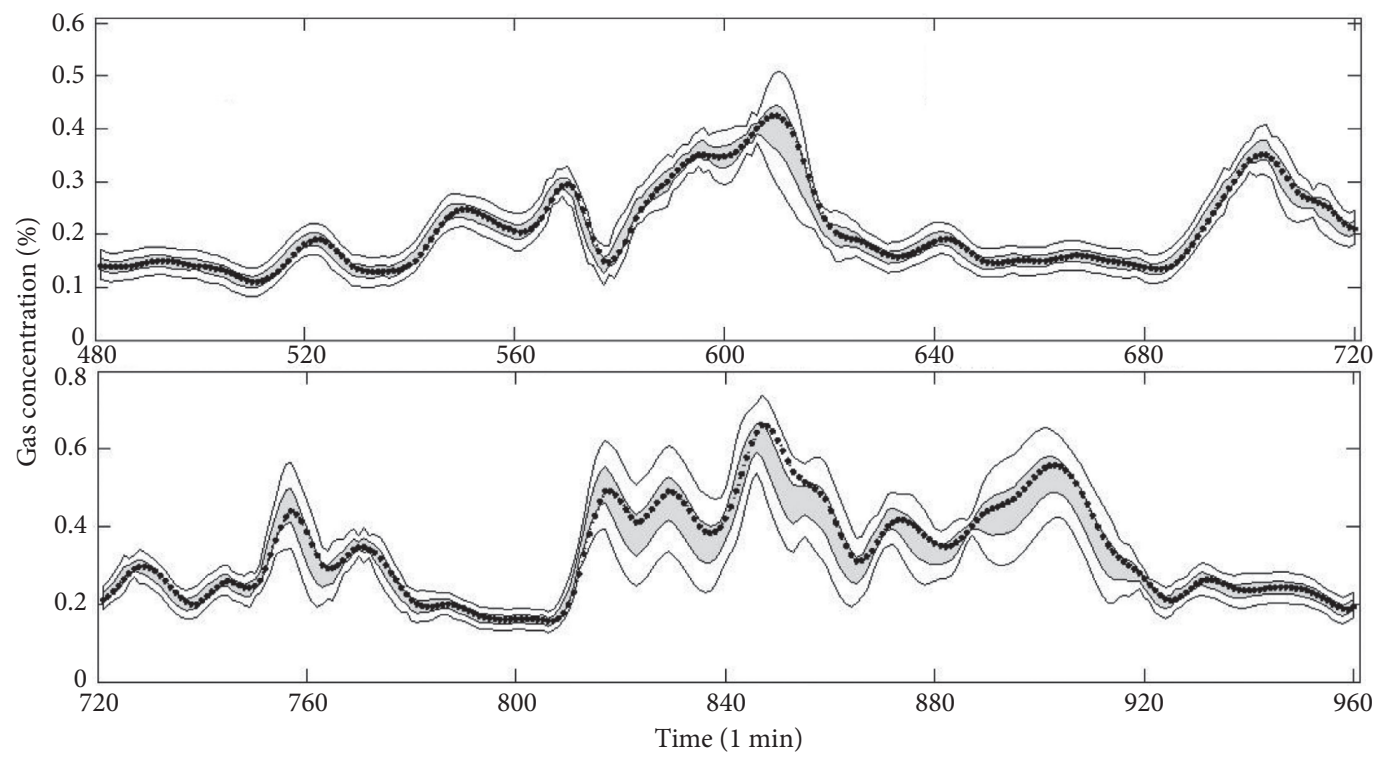

99.73\% confidence interval

$\square 8.27 \%$ confidence interval

-. Actual monitoring value

(b)

Figure 5: Continued. 


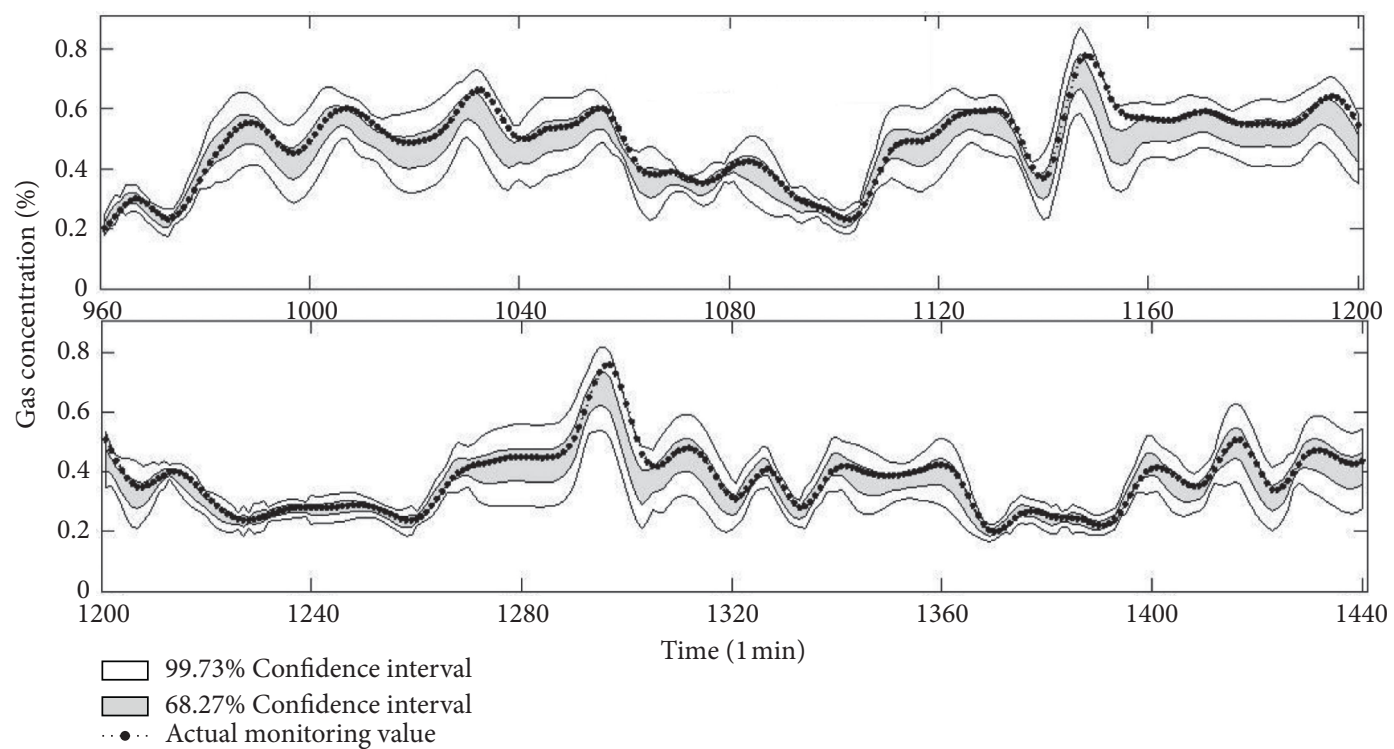

(c)

FIGURE 5: GPR prediction results of gas concentration at the working face monitoring point.

information of the gas concentration variation trend of the working face monitoring point. By calculating the correlation degree of the confidence interval, the relationship between the average correlation degree and average reliability and average interval length is obtained, which is the basis for obtaining the optimal confidence interval. The calculation results of historical data show that when $\rho=0.6$, the correlation degree and reliability of confidence interval reach the maximum value. Therefore, under the condition of monitoring failure in the working face, the best resolution coefficient for correlation analysis of confidence interval in multiple monitoring points is $\rho=0.6$ in this time period.

In practical application, with the continuation of mining process, the optimal value of $\rho$ should be calculated dynamically to adapt to the variation of gas concentration caused by the variation of geological conditions and production process.

\subsection{Decision-Making Optimization of Gas Concentration} Monitoring. Based on the optimal resolution coefficient $\rho$, the dynamic calculation and optimization of the confidence interval are carried out by adjusting the confidence coefficient $\beta$. The comparison between the optimal confidence interval of gas concentration and the initial confidence interval at the working face monitoring point on January 1, 2018, is performed and shown in Figure 7.

In Figure 7, when adjusting $\beta=1.72$ (91.46\% confidence interval), the optimal confidence interval is abstained. The first 8 hours' (preparation shift) actual monitoring values all fall within the confidence interval; in the second and the third 8 hours (production work shifts), only 12 points fall outside the confidence interval; the reliability is $98.75 \%$, the average confidence interval length is $0.069 \%$, and the average gas concentration is $0.51 \%$, and the ratio of them is $13.52 \%$, which is in the reasonable error range of gas monitoring. Therefore, in practical application, the calibration value of gas sensor can meet the requirements if it falls in the confidence interval, or it can be closer to the actual monitoring value when the median value of the confidence interval is directly taken as the calibration value.

Compared with other representative methods that use mine gas monitoring data to realize gas concentration prediction, the main methods in relevant literature are used to make interval prediction for the sample data in this section, and the average reliability of confidence interval and the average interval length are calculated. The calculation results are shown in Table 2 .

It can be seen from Table 2 that the results of this research are better than those of other methods. Compared with other methods, this research makes full use of the effective monitoring information of real-time monitoring big data from multiple monitoring points; not only does it have high confidence interval reliability, but also the interval length obtained after optimization calculation is small, which is more suitable for practical application. The main advantages are that the normal distribution interval number predicted by GPR has good probability characteristics, which provides a basis for further decision-making optimization, and the dynamic adjustment of model parameters in the correlation analysis model avoids the subjective defects in grey correlation analysis and ensures the feasibility and applicability of of this method in actual application. 


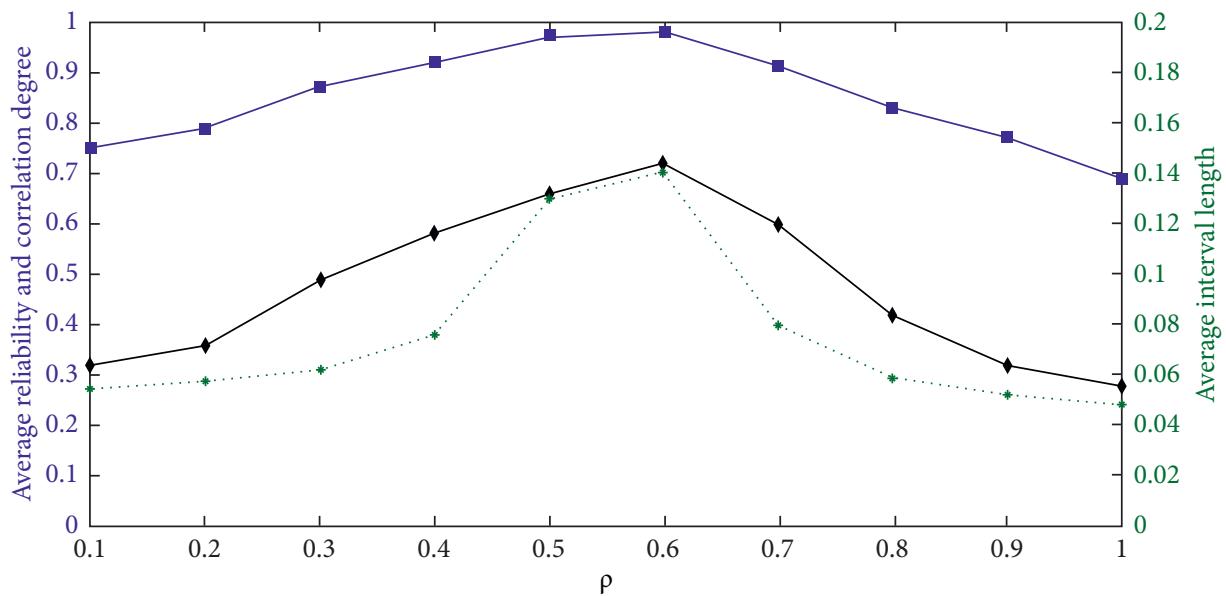

$\rightarrow-$ Average reliability

$\rightarrow$ Average correlation degree

*.. Average interval length

Figure 6: The correlation analysis results of interval number.

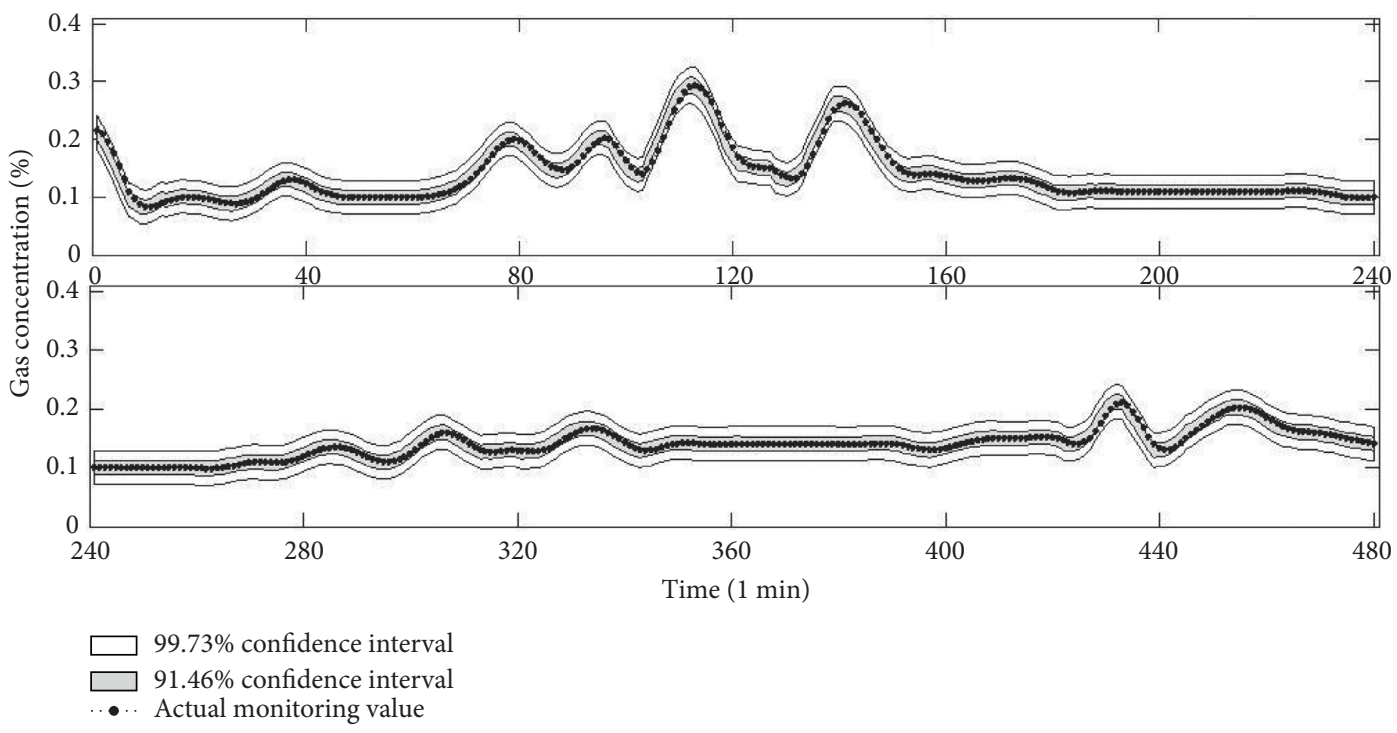

(a)

Figure 7: Continued. 


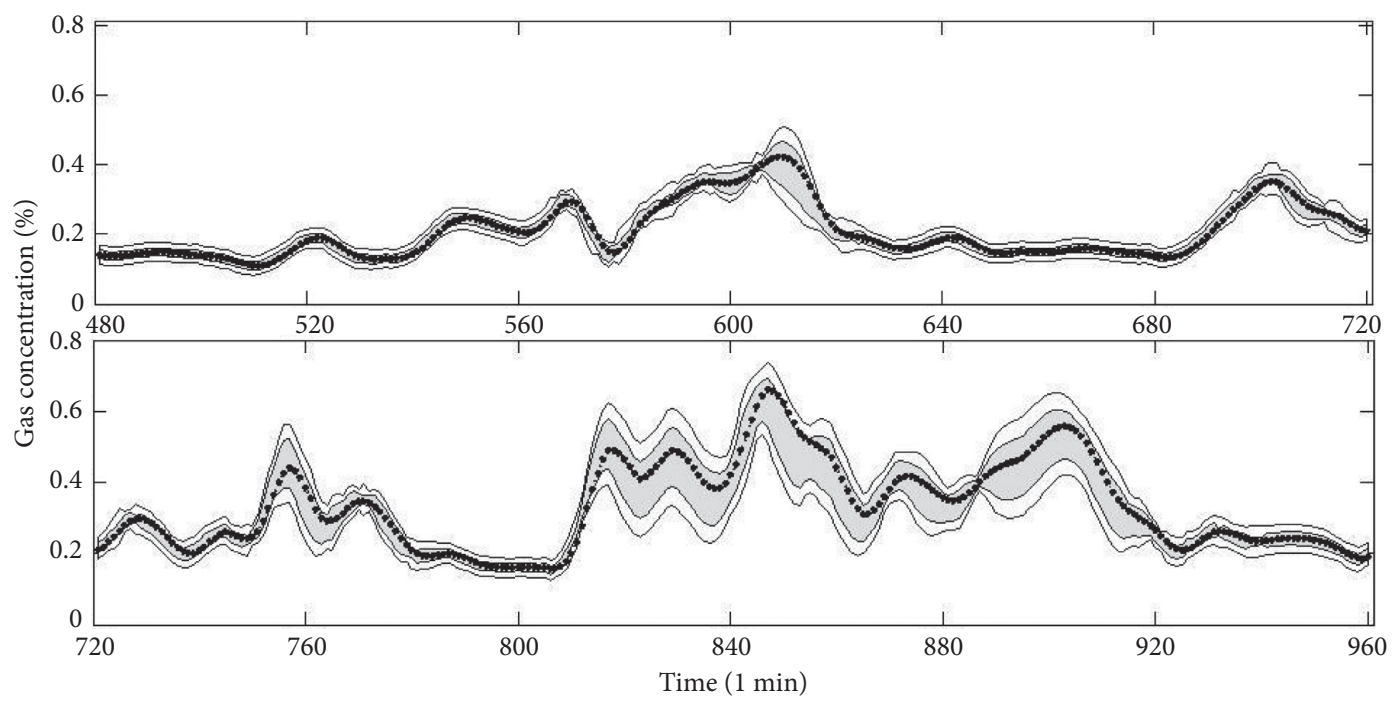

99.73\% Confidence interval

$\square$ 91.46\% Confidence interval

-. Actual monitoring value

(b)

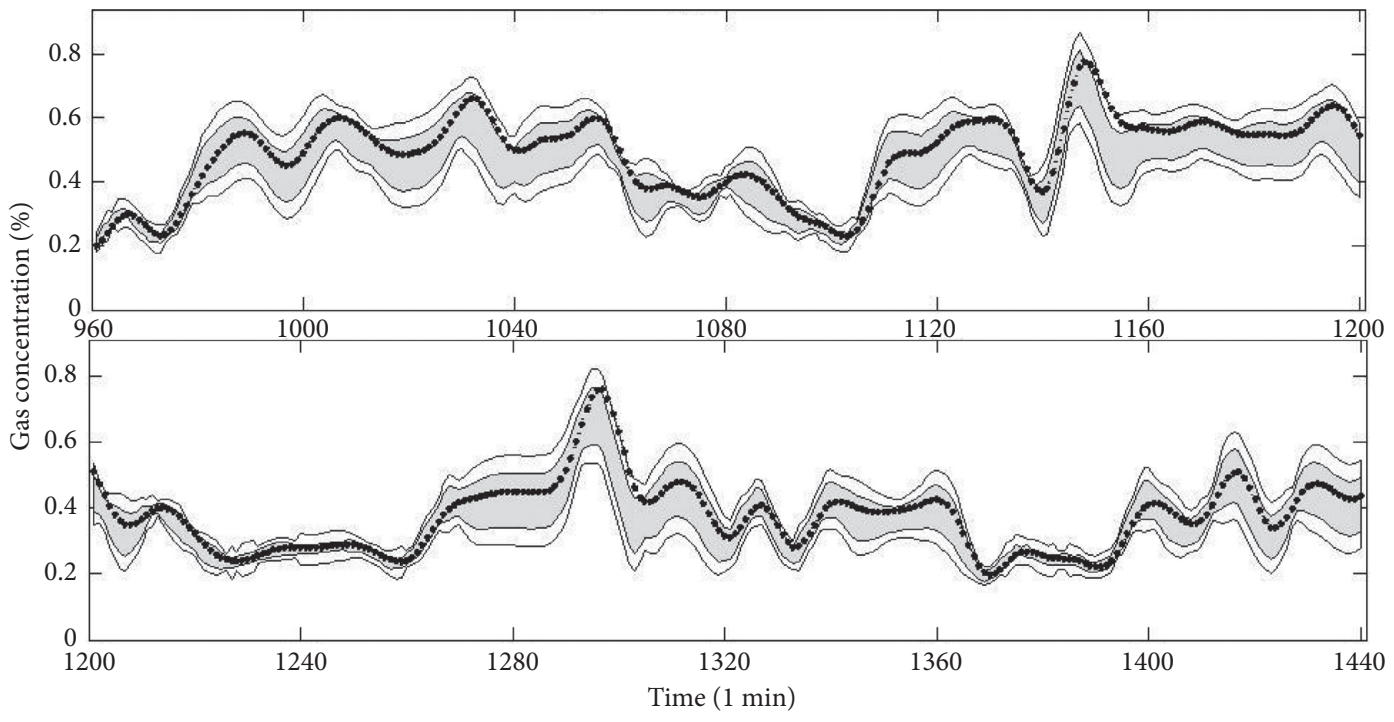

99.73\% Confidence interval

91.46\% Confidence interval

-. Actual monitoring value

(c)

Figure 7: The optimizing results for confidence interval of gas concentration.

TABLE 2: Comparison with other methods.

\begin{tabular}{lccc}
\hline Relevant research & Methods for comparative analysis & Average reliability (\%) & Average interval length \\
\hline$[20]$ & Chaotic time series model & 69.9 & 0.128 \\
{$[21]$} & Support vector machine regression & 86.6 & 0.091 \\
{$[22]$} & Grey interval prediction & 62.4 & 0.144 \\
{$[23]$} & Neural network model & 78.1 & 0.102 \\
{$[24]$} & Fuzzy information granulation and Markov correction model & 76.5 & 0.109 \\
& This research method & 99.8 & 0.069 \\
\hline
\end{tabular}




\section{Conclusion}

The GPR prediction model fully considers various monitoring failures and integrates the effective information of monitoring data at multiple monitoring points in the working face area. By taking advantage of effective information in the data of associated monitoring points, it obtains the reliable initial confidence interval under the condition of monitoring failure; by determining the reasonable confidence coefficient, the initial confidence interval can contain the actual monitoring value of gas concentration as far as possible, and the reliability of subsequent decision-making calculation can be ensured.

In the correlation analysis of interval number, the determination of resolution coefficient directly affects the length of confidence interval; its dynamic adjustment avoids the subjectivity of similar methods, and we come to the important conclusion that the optimal value of resolution coefficient corresponds to the optimal confidence interval. The optimal confidence interval not only reduces the length of interval but also contains the actual monitoring value with great probability, so it can be used to assist sensor calibration effectively.

The method proposed in this paper has been verified by online calculation. The optimized confidence interval meets the accuracy and reliability requirements of sensor calibration decision-making in coal mines, so this method can be developed by programming and deployed in portable devices, which can provide convenient and effective auxiliary means for technicians to carry out the daily gas sensor calibration.

\section{Data Availability}

The data used to support the findings of this study have not been made available because the data in case study originated from the safety monitoring and monitoring system of Huangling No. 2 coal mine in Shaanxi Province in China.

\section{Ethical Approval}

This work only involved computer simulations of engineering problem and did not involve any active collection of human data.

\section{Conflicts of Interest}

The author declares that there are no conflicts of interest.

\section{Acknowledgments}

This work has been supported by the National Natural Science Foundation of China (Grant 51734007).

\section{References}

[1] C. E. Rasmussen, "Advances in Gaussian processes," in Proceedings of the Neural Information Processing Systems Conference, The MIT Press, Vancouver, Canada, 2006.
[2] J. Kocijan, Modelling and Control of Dynamic Systems Using Gaussian Process Models, Springer, Berlin, Germany, 2016.

[3] G. Gregorčič and G. Lightbody, "Gaussian process approach for modelling of nonlinear systems," Engineering Applications of Artificial Intelligence, vol. 22, no. 4-5, pp. 522-533, 2009.

[4] G. Gregorčič and G. Lightbody, "Nonlinear system identification: from multiple-model networks to Gaussian processes," Engineering Applications of Artificial Intelligence, vol. 21, no. 7, pp. 1035-1055, 2008.

[5] G. Pillonetto and G. De Nicolao, "A new kernel-based approach for linear system identification," Automatica, vol. 46, no. 1, pp. 81-93, 2010.

[6] J. Ko and D. Fox, "GP-BayesFilters: bayesian filtering using Gaussian process prediction and observation models," $\mathrm{Au}$ tonomous Robots, vol. 27, no. 1, pp. 75-90, 2009.

[7] D. W. Dong, S. G. Li, X. T. Chang, and H. F. Lin, "Prediction model of gas concentration around working face using multivariate time series," Journal of Mining \& Safety Engineering, vol. 29, no. 1, pp. 135-139, 2012.

[8] B. Ni, Z. Y. Liu, and G. Yu, "Ultrasound image segmentation method based on Gaussian process and deformable model," Computer Engineering and Applications, vol. 52, no. 12, pp. 210-215, 2016.

[9] Z. J. Feng and Y. Fan, "Chaotic time series prediction based on Gaussian processes mixture," Journal of System Simulation, vol. 31, no. 7, pp. 1387-1396, 2019.

[10] T. J. Tian, C. R. Lu, and H. J. Huang, "Study on the route choice behaviors based on interval travel and reference points," Journal of Systems Science and Mathematical Sciences, vol. 38, no. 4, pp. 395-405, 2018.

[11] Z. H. Zhang, "Fast multiobjective immune optimization approach solving multiobjective interval number programming," Swarm and Evolutionary Computation, vol. 59, pp. 1-22, 2019.

[12] J. J. Wang, F. He, and Z. X. Wu, "Interval quadratic programming model for portfolio selection with improved interval acceptability degree," Chinese Journal of Management Science, vol. 26, no. 9, pp. 11-18, 2018.

[13] J. H. Li, H. Y. Yu, Y. J. Wu, and L. Ming, "Research on laser optimum work area of resonator fiber optical gyroscope," Navigation Positioning \& Timing, vol. 4, no. 6, pp. 107-112, 2017.

[14] L. Q. Jin, X. Fang, and Y. Xu, "Multi-attribute decision making factor based on evidential reasoning and prospect theory," Fuzzy Systems and Mathematics, vol. 31, no. 6, pp. 124-131, 2017.

[15] X. N. Wu, Q. Z. Wang, C. Y. Zhang, and Z. Ye, "Trust evaluation method of cloud service based on interval multiple attribute decision making," Journal of Chinese Computer Systems, vol. 39, no. 7, pp. 1492-1497, 2018.

[16] G. Q. Huang, L. M. Xiao, and G. B. Zhang, "Improved failure mode and effect analysis with interval-valued intuitionistic fuzzy rough number theory," Engineering Applications of Artificial Intelligence, vol. 95, pp. 1-17, 2020.

[17] S.-P. Wan, F. Wang, G.-L. Xu, J.-Y. Dong, and J. Tang, “An intuitionistic fuzzy programming method for group decision making with interval-valued fuzzy preference relations," Fuzzy Optimization and Decision Making, vol. 16, no. 3, pp. 269-295, 2017.

[18] S. Wan, F. Wang, and J. Dong, "A group decision making method with interval valued fuzzy preference relations based on the geometric consistency," Information Fusion, vol. 40, pp. 87-100, 2018. 
[19] X.-J. Cheng, S.-P. Wan, and J.-Y. Dong, "A new consistency definition of interval multiplicative preference relation," Fuzzy Sets and Systems, vol. 409, pp. 55-84, 2021.

[20] B. Y. Zhang, R. Li, and W. Y. Mu, "Study on gas concentration prediction based on chaotic time series," Computer Engineering and Applications, vol. 47, no. 10, pp. 244-248, 2011.

[21] M. Y. Qiao, X. P. Ma, J. Y. Lan, and Y. Wang, "Time-series gas prediction model using LS-SVR within a Bayesian framework," Mining Science and Technology, vol. 21, no. 1, pp. 153-157, 2011.

[22] D. Huang, X. B. Xie, and X. Y. Huang, "Forecasting method of coalmine gas concentration based on grey self-memorization theory," Science \& Technology Review, vol. 23, no. 17, pp. 58-62, 2010.

[23] S. G. Li, L. Ma, S. B. Pan, and X. Shi, "Research on prediction model of gas concentration based on RNN in coal mining face," Coal Science and Technology, vol. 48, no. 1, pp. 33-37, 2020.

[24] Y. M. Wu, C. R. Qiu, and X. B. Liu, "Prediction of gas concentration based on fuzzy information granulation and Markov correction," Coal Technology, vol. 37, no. 5, pp. 172-175, 2018. 